\title{
Complementary DNA Cloning of HSC71, a 71 kDa Heat Shock Cognate Protein, in Japanese Flounder Paralichtys olivaceus
}

\author{
Yoshihiro Yokoyama, ${ }^{* 1, \dagger}$ Hisashi Hashimoto, ${ }^{* 2}$ Satoshi Kubota, ${ }^{* 2}$ \\ Masato Kinoshita, ${ }^{* 2}$ Haruhiko Toyohara, ${ }^{* 2, \dagger}$ \\ Morihiko Sakaguchi, ${ }^{* 2}$ and Masao Kanamori ${ }^{* 1}$ \\ *IInterdisciplinary Research Institute for Biosciences, Mukogawa Women's University, Ikebiraki, \\ Nishinomiya, Hyogo 663-8558, Japan \\ ${ }^{*}$ Division of Applied Biosciences, Graduate School of Agriculture, Kyoto University, Sakyo-ku, \\ Kyoto 606-8224, Japan
}

(Received September 22, 1997)

Full-length cDNA for a $71 \mathrm{kDa}$ constitutively expressed heat-shock-cognate protein (HSC71), a member of the HSP70 family, was isolated from a Japanese flounder embryo cDNA library. It has a single ORF of $1950 \mathrm{bp}$ that encodes a protein of $71.2 \mathrm{kDa}$. Japanese flounder HSC71 contains a EEVD (Glu-Glu-Val-Asp) peptide motif at the C-terminal end which is a common feature of cytosolic HSC proteins in the HSP70 family. Japanese flounder HSC71 is $96.0 \%, 93.9 \%, 93.5 \%$, and $93.4 \%$ identical in primary structure to rainbow trout HSC71, bovine HSC71, rat HSC71, and human HSC71, respectively. These results suggest that the HSC71 amino acid sequence has been highly conserved among vertebrates. Northern blot analysis showed that HSC71 mRNA was expressed constitutively in the Japanese flounder cells in early passage regardless of heat shock treatment.

Key words: HSC71, heat shock cognate protein, cDNA, Japanese flounder

All organisms synthesize a few evolutionary-conserved proteins called heat shock proteins or HSP, recently called a stress protein, in response to various harmful environmental stresses. ${ }^{1)}$ The most abundant and conserved HSP has a molecular mass of about $70 \mathrm{kDa}$. In eukaryotes, HSP70 are encoded by heat-inducible HSP70 genes and usually their basal expression is negligible. Several homologous were subsequently discovered, which were constitutively expressed under normal and stress conditions in contrast to HSP70. ${ }^{1)}$ One of these so-called heat shock cognate genes encodes about $70 \mathrm{kDa}$ protein (HSC70) which is particularly abundant in embryos and ovaries. ${ }^{2)}$ In yeast, genetic analysis has shown that double mutations into two cognate genes affected the growth rate at normal temperature, but not at a high temperature which induces the expression of HSP70, ${ }^{3}$ suggesting that HSC70 is essential for normal growth. Although the patterns in expression of HSP and HSC are different, it was shown that the HSC70 and HSP70 have similar structure and function as molecular chaperones in cultured cells of mammals. ${ }^{4)}$ These findings suggest that HSC70 is indispensable for the development of fish embryos under normal and stress conditions.

Japanese flounder is one of the most economically important fish in Japan. The cultivation of Japanese flounder has been successful. During the transportation and breeding in the farm, the embryos could be exposed to adverse environmental changes. Therefore, stress proteins includ- ing HSC70 may have important roles for the development under normal and stress conditions. In fact, in vitro studies revealed that the cultured fish cells constitutively synthesized HSC70 under normal conditions and adverse conditions such as heat shock, heavy metal ions, and sodium arsenite. ${ }^{1)}$ However, little is known about such proteins and those gene structures in Japanese flounder. As a preliminary experiment to resolve the role of stress protein in the flounder embryos, we decided to clone the HSC70related cDNA, referred to here as Japanese flounder HSC71 cDNA. Nucleotide sequence and expression analyses revealed that the isolated clone represents a heat shock cognate 70 cDNA.

\section{Materials and Methods}

\section{Japanese Flounder Embryos and Culture of cells}

Japanese flounder Paralichtys olivaceus embryos in the eyed period were kindly supplied by Yamagataya (Nantan, Hyogo, Japan). Primary cultured cells of Japanese flounder embryos were kindly donated by Dr. T. Seikai (Fisheries Research Station, Faculty of Agriculture, Kyoto University). The cells were cultured at $25^{\circ} \mathrm{C}$ in air using Leibovitz's L15 medium supplemented with $25 \mathrm{~mm}$ 4-(2hydroxyethyl)-1-piperazine-ethanesulfonic acid, penicillin potassium (200 international units $/ \mathrm{m} l$ ), streptomycin sulfate $(200 \mu \mathrm{g} / \mathrm{m} l)$, and $10 \%$ fetal bovine serum (Biological

\footnotetext{
${ }^{\dagger}$ To whom correspondence should be addressed. E-mail: yokoyama@mwu.mukogawa-u.ac.jp or toyohara@kais.kyoto-u.ac.jp

The following abbreviations are used: HSC, heat shock cognate protein(s); HSP, heat shock protein(s); RT-PCR, reverse transcription-polymerase chain reaction; cDNA, DNA complementary to mRNA; bp, base pair; kb, kilobase; dNTP, deoxynucleotide triphosphate; SDS, sodium dodecyl sulphate; SSC, standard sodium chloride/sodium citrate buffer

The DDBJ, GenBank and EMBL accession number for Japanese flounder HSC71 is AB006814.
} 
Industries).

\section{Preparation of $R N A$}

Total RNA from embryos of Japanese flounder or the cultured cells in early passage from flounder embryo was isolated by ultracentrifugation in $5.7 \mathrm{M}$ cesium chloride after homogenization in $4 \mathrm{M}$ guanidinium thiocyanate. ${ }^{5}$ Poly (A) + RNA was enriched by chromatography on oligo (dT) cellulose (Collaborative Biomedical Products) or on Oligotex-dT30 (Takara).

\section{$R T-P C R$ Cloning}

To obtain a partial sequence of flounder HSC71 gene, PCR was conducted with degenerate oligonucleotide primers having the following sequences: $5^{\prime}$-ATCGAYCTSGGSACYACCTACTC-3' as the sense primer and $5^{\prime}$-GCACCGTASSCSACCGCYTCRTC-3' as the an- tisense primer, where $\mathrm{R}=\mathrm{A}+\mathrm{G}, \mathrm{S}=\mathrm{G}+\mathrm{C}, \mathrm{Y}=\mathrm{C}+\mathrm{T}$. These correspond to the amino acid sequences IDLGTTYS and DEAVAYGA, respectively, which are identical in rainbow trout HSC71, ${ }^{6}$ human HSC $71,{ }^{7}$ (shown in Fig. 1), bovine $\mathrm{HSC} 71,{ }^{8)}$ and rat $\mathrm{HSC} 71 .{ }^{9)}$ Single-stranded cDNA was synthesized from $1 \mu \mathrm{g}$ of total RNA of the embryo with 200 units of MMLV-RT (Gibco-BRL) in a $20 \mu l$ reaction mixture comprising $50 \mathrm{mM} \mathrm{KCl}, 20 \mathrm{mM}$ Tris- $\mathrm{HCl}$ (pH 8.4), $2.5 \mathrm{mM} \mathrm{MgCl}_{2}, 0.1 \mathrm{mg} / \mathrm{m} l \mathrm{BSA}, 1 \mathrm{mM}$ each $\mathrm{dNTP}, 5 \mu \mathrm{M}$ random hexamer, and 20 units of ribonuclease inhibitor RNasin (Toyobo). A quarter of the resultant cDNA was used as a template for the PCR, which was carried out in a $25 \mu l$ solution comprising 8 units of $\triangle$ Tth DNA polymerase (Toyobo), $50 \mathrm{mM} \mathrm{KCl,} 20$ $\mathrm{mM}$ Tris- $\mathrm{HCl}$ ( $\mathrm{pH} 8.4$ ), $2.5 \mathrm{mM} \mathrm{MgCl}_{2}, 0.1 \mathrm{mg} / \mathrm{ml}$ BSA, $0.2 \mathrm{~mm}$ each dNTP, and $0.2 \mu \mathrm{M}$ each primer. The PCR conditions were $30 \mathrm{~s}$ at $95^{\circ} \mathrm{C}, 1 \mathrm{~min}$ at $56^{\circ} \mathrm{C}$, and $2 \mathrm{~min}$ at

$-61$

gacaagtccgcctcaccgcacccaacacctaaagtcatcagcggttcaattatccggaatc

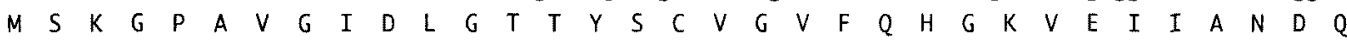
1 ATGTCTAAGGGACCAGCAGTTGGTATTGATCTCGGGACCACCTACTCCTGTGTTGGTGTGTTCCAGCATGGAAAAGTTGAGATCATTGCCAATGACCAGG 100

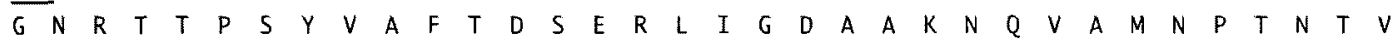
101 GCAACAGGACCACACCCAGCTATGTGGCCTTCACAGATTCCGAGAGGCTGATTGGAGATGCAGCCAAAAACCAGGTTGCCATGAACCCCACCAACACGGT Z00

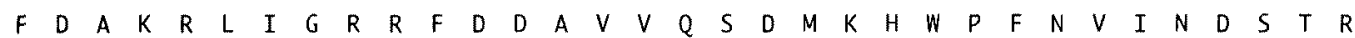
201 CTTGATGCCAAAAGGCTGATCGGCCGCAGGTTCGATGACGCAGTTGTACAATCAGATATGAAGCACTGGCCATTCAATGTCATCAATGACAGCACTCGC 300

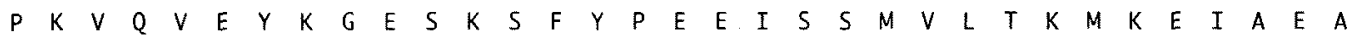

301 CCCAAAGTTCAAGTTGAGTACAAGGGTGAGTCAAAGTCCTTCTACCCAGAGGAGATCTCATCCATGGTGCTGACCAAGATGAAGGAGATTGCTGAAGCCT 400

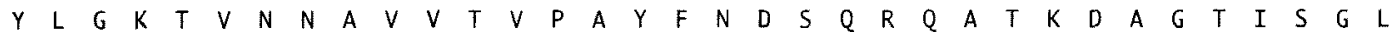
401 ACCTCGGAAAGACTGTCAACAATGCTGTAGTCACAGTGCCCGCCTACTTCAATGACTCCCAGCGCCAGGCCACCAAGGATGCAGGCACTATCTCTGGCCT 500

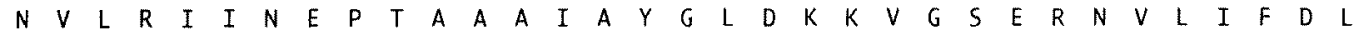
501 CAATGTTTTGCGTATCATCAATGAACCGACTGCTGCTGCCATCGCCTATGGTTTGGACAAGAAGGTTGGATCAGAAAGGAACGTCCTCATCTTCGATCTT 600

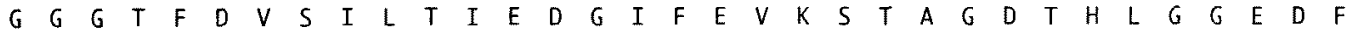
601 GGTGGTGGCACCTITGATGTGTCCATCTTGACCATTGAGGATGGCATCTTTGAGGTCAAGTCCACCGCTGGAGATACTCATCTTGGTGGGGAGATTTCG 700

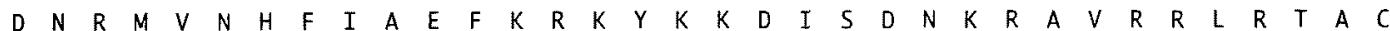
701 ACAACCGCATGGTCAACCACTICATCGCTGAGTTCAAGCGCAAGTACAAGAAAGACATCAGCGACAACAAGAGAGCTGTCCGTCGTCTGCGCACCGCTTG 8O0

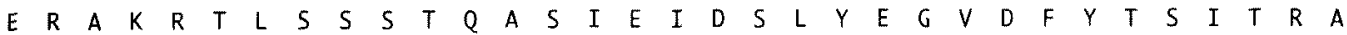
801 TGAGAGGGCAAAGCGCACATTGTCTTCCAGCACCCAGGCCAGCATCGAAATCGACTCCCTGTATGAGGGAGTTGACTTITACACCTCCATCACCAGGGCT 900 $R$ F E E L N N A D $L$ 901 CGCTTTGAGGAGCTCAATGCTGACCTCTTCCGTGGCACACTAGACCCTGTGGAGAAGTCTCTCCGTGATGCCAAGATGGATAAGGGACAGATCCATGACA 1000

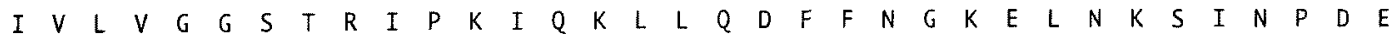
1001 TTGTCCTGGTCGGTGGCTCCACCCGTATCCCCAAGATCCAGAAGCTGCTCCAGGACTTCTTCAACGGAAAGGAGCTCAACAAGAGTATCAACCCTGATGA 1100

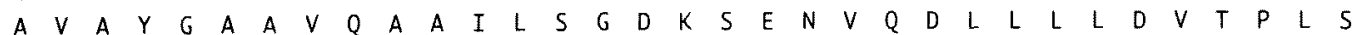

1101 AGCTGTGGCCTATGGAGCCGCTGTCCAGGCTGCCATCCTGTCTGGTGACAAGTCTGAAAATGTGCAGGACCTGCTGCTTCTGGATGTCACCCCCCTGTCC 1200

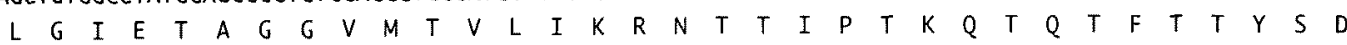

1201 CTGGGTATTGAGACTGCTGGAGGCGTCATGACTGTCTTGATCAAACGCAACACCACCATTCCTACTAAGCAGACCCAGACCTTTACCACCTACTCTGACA 1300

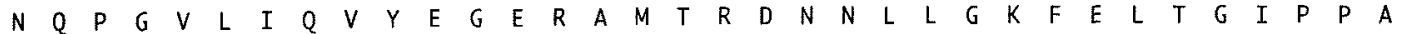
1301 ACCAGCCTGGTGTGCTCATCCAGGTTTATGAGGGTGAGCGTGCCATGACCAGAGACAACAACCTGCTGGGCAAGTTTGAGCTGACAGGCATCCCTCCTGC 1400

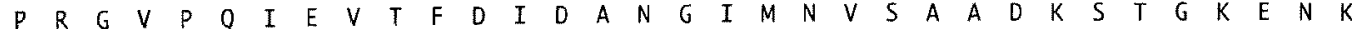
1401 TCCTCGTGGTGTTCCCCAGATCGAGGTGACATTTGATATCGATGCCAACGGCATCATGAATGTCTCTGCTGCTGACAAGAGCACTGGAAAGGAGAACAAG 1500

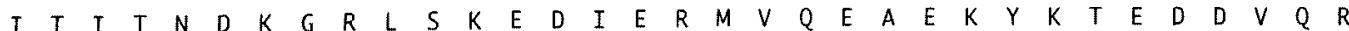
1501 ATCACCATCACCAATGACAAGGGTCGTCTCAGCAAGGAGGACATTGAACGCATGGTCCAGGAGGCTGAGAAGTACAAGACAGAGGATGATGTCCAGCGTG 1600

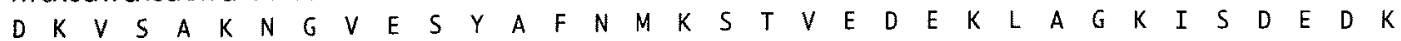
1601 ACAAGGTGTCTGCAAAGAATGGAGTGGAGTCATATGCTTTCAACATGAAGTCAACTGTGGAGGATGAGAAGCTCGCTGGCAAGATCAGTGATGAAGATAA 1700

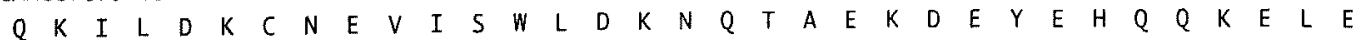

1701 GCAGAAGATCTTGGACAAGTGCAACGAGGTCATCAGCTGGCTGGACAAGAACCAGACTGCCGAAAAGGATGAATATGAGCATCAACAAAAGGAACTGGAG 1800 $K V G N$ P I I T K L Y Q S A G G M P G G M P E G M P G G F G G A G

1801 AAGGTGTGCAACCCCATCATCACTAAGCTGTACCAGAGTGCCGGTGGCATGCCAGGTGGTATGCCAGAAGGCATGCCTGGTGGCTTCGGTGGAGCTGGTG 1900 G A A P G A G S S G P $T$ T I $E$ E $E$ V

1901 GTGCCGCTCCTGGCGCTGGATCATCTGGCCCCACCATTGAGGAGGTCGACtaaacattccetcagctgetacctcctgagaaagtgctctaagaaagtaa 2000 2001 ccctctatagcacaagttacaagcttaagagtgtaatttaaagtatcttgttattgcatcatttgacacaactgggaacagcttgtacttggctcagtg 2100 2101 gagcagtgagtcacaaagcgttttgtatgatgtcactgccttgtcaataaacatgaaaccetcaaaaaaaaaaaaaaaaaa 2181

Fig. 1. Nucleotide and deduced amino acid sequences of the HSC71 cDNA from Japanese flounder embryos.

Small letters denote the $5^{\prime}$ and $3^{\prime}$ untranslated regions and capital letters denote the coding regions. The position of initiation (base pairs 1 to 3 ), termination (1951 to 1953) codons, Eco RV (1435 to 1440) and HindIII (2022-2027) sites, and polyadenylation signal (2146 to 2151$)$ in the sequence are underlined. 
$72^{\circ} \mathrm{C}$ for 25 cycles (Astec, Program Temp Control System PC-700). The resultant products of the expected size, approximately $1090 \mathrm{bp}$, were subcloned in pBluescript II KS(Stratagene). DNA sequencing analysis revealed that there was a cDNA fragment of HSC71-like gene in the pool of the PCR products.

\section{cDNA Cloning and Sequencing}

The synthesis of double-stranded cDNA was accomplished with a ZAP-cDNA Synthesis Kit (Stratagene) using poly (A) + RNA prepared from embryo by chromatography on oligo (dT) cellulose. The cDNAs were sizefractionated on a CHROMA SPIN-100 column (Clontech) and then adapter-ligated prior to the generation of an oligo (dT) primed library in Uni-ZAP/Eco RI/Xho I/CIAP (Stratagene). The cDNA library was screened with a
cDNA fragment obtained on PCR as a probe. Plaque hybridization was performed at $42^{\circ} \mathrm{C}$ in a solution comprising $5 \times \mathrm{SSC}(1 \times \mathrm{SSC}=150 \mathrm{mM} \mathrm{NaCl}, 15 \mathrm{~mm}$ sodium citrate, $\mathrm{pH} 7.0$ ), $0.1 \%$ SDS, $50 \%$ formamide, $100 \mathrm{mg} / \mathrm{ml}$ denatured salmon sperm DNA, 0.6\% Ficoll 400, 0.6\% polyvinylpyrrolidone $100,0.6 \% \mathrm{BSA}$, and ${ }^{32} \mathrm{P}$-labeled probe prepared with a Megaprime DNA labelling system (Amersham International PLC). After hybridization, the filters were washed four times with $2 \times \mathrm{SSC}$ at room temperature and three times with $2 \times \mathrm{SSC}, 0.5 \%$ SDS at $65^{\circ} \mathrm{C}$ prior to autoradiography. Plasmids (pBluescript SK-) containing the cDNA insert were obtained by in vivo excision from positive single plaques according to the manufacturer's instruction (Stratagene). After subcloning into pBluescript II KS+, DNA fragments were sequenced by a PCR procedure employing fluorescent dideoxynucleotides

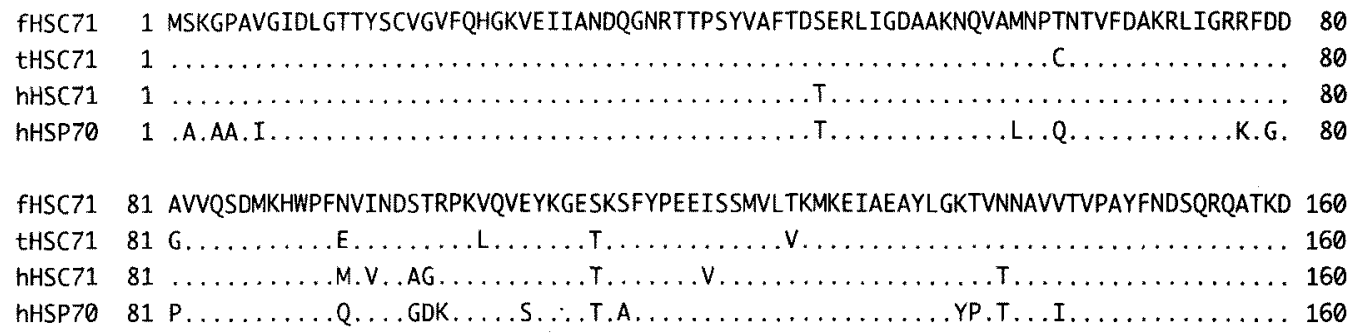

fHSC71 81 AVVQSDMKHWPFNVINDSTRPKVQVEYKGESKSFYPEEISSMVLTKMKETAEAYLGKTVNNAVVTVPAYFNDSQRQATKD 160

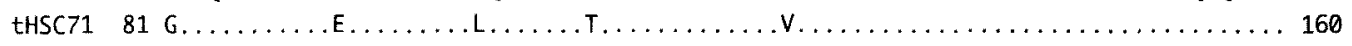

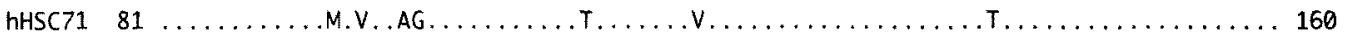

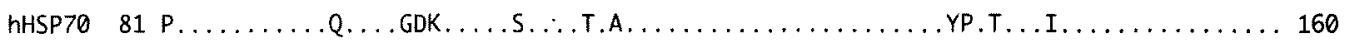

fHSC71 161 AGTISGLNVLRIINEPTAAAIAYGLDKKVGSERNVLIFDLGGGTFDVSILTIEDGIFEVKSTAGDTHLGGEDFDNRMVNH 240

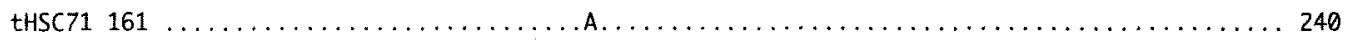

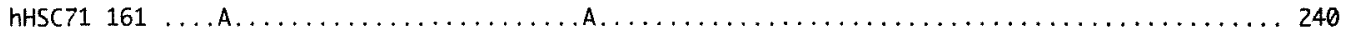

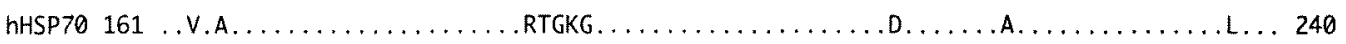

fHSC71 241 FIAEFKRKYKKDISDNKRAVRRLRTACERAKRTLSSSTQASIEIDSLYEGVDFYTSITRARFEELNADLFRGTLDPVEKS 320

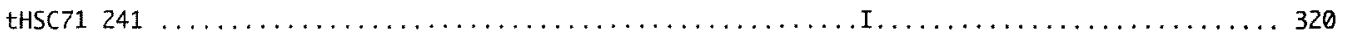

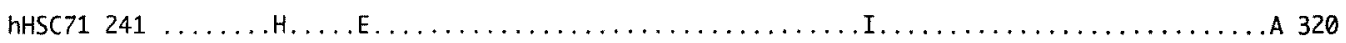

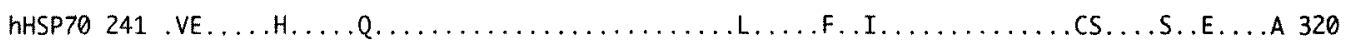

FHSC71 321 LRDAKMDKGQIHDIVLVGGSTRIPKIQKLLQDFFNGKELNKSINPDEAVAYGAAVQAAILSGDKSENVQDLLLLDVTPLS 400

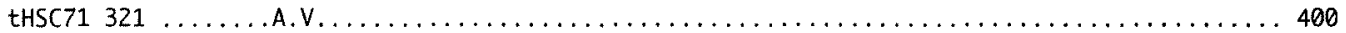

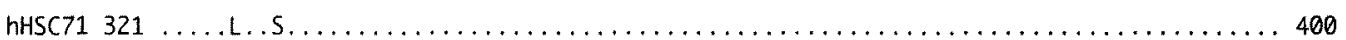

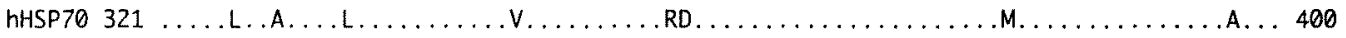

fHSC71 401 LGIETAGGVMTVLIKRNTTIPTKQTQTFTTYSDNQPGVLIQVYEGERAMTRDNNLLGKFELTGIPPAPRGVPQIEVTFDI 480

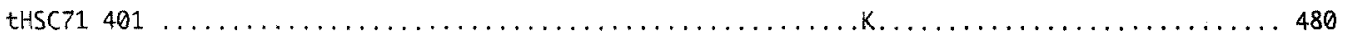

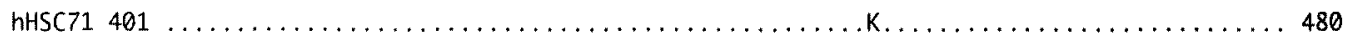

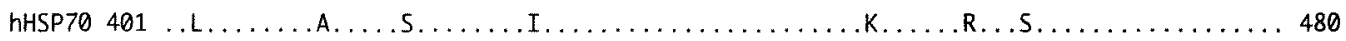

fHSC71 481 DANGIMNVSAADKSTGKENKITITNDKGRLSKEDIERMVQEAEKYKTEDDVQRDKVSAKNGVESYAFNMKSTVEDEKLAG 560

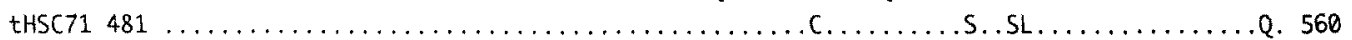

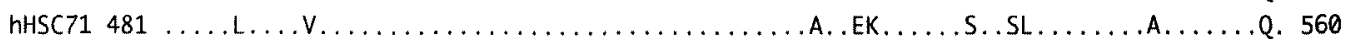

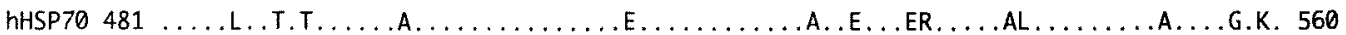

fHSC71 561 KISDEDKQKILDKCNEVISWLDKNQTAEKDEYEHQQKELEKVCNPIITKLYQSAGGMPGGMPEGMPGGFGGAGGAAPGAG 640

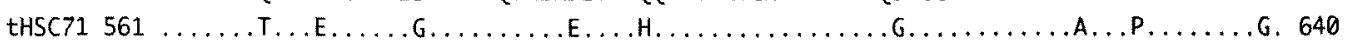

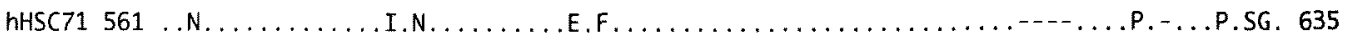

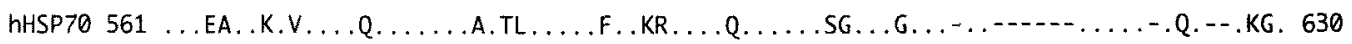
$\begin{array}{llll}\text { FHSC71 } 641 & \text {-SSGPTIEEVD } & 650\end{array}$ tHSC71 $641 \mathrm{G} \ldots \ldots \ldots \ldots .651$ hHSC71 561 A....... hHSP70 631 SG, .....

Fig. 2. Protein comparison.

The deduced amino acid sequence of a protein encoded by the cloned Japanese flounder cDNA (fHSC71) is compared to that of rainbow trout constitutively synthesized HSC71 (tHSC71), ${ }^{6}$ human HSC71 (hHSC71), ${ }^{7}$ and human heat-inducible HSP70 (hHSP70). 10) Only different amino acids are indicated and hyphens mark deletions. 


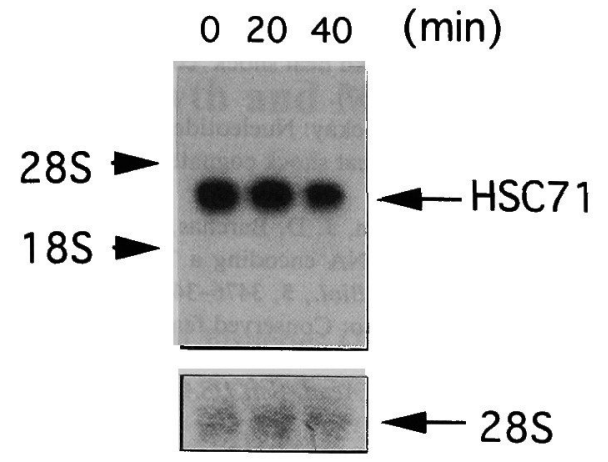

Fig. 3. RNA expression analysis.

Northern blot analyses were carried out using $10 \mu \mathrm{g}$ total RNA isolated from control $(0 \mathrm{~min})$ and heat-shocked $\left(37^{\circ} \mathrm{C}\right.$ for 20 or $\left.40 \mathrm{~min}\right)$ Japanese flounder cells in early passage. The probe for hybridization was prepared from the cloned cDNA fragments (base pairs 1438 to 2026). Hybridization was done under the same conditions as described above. As an internal control, 28S rRNA on the filter was stained with methylene blue after hybridization and exposure to $\mathrm{X}$ ray film.

and a model 373A automated sequencer (Applied Biosystems Inc.). The sequences reported in this paper were determined on both strands.

\section{Northern Blot Analysis}

The cultured cells from Japanese flounder embryos in early passage were heat-shocked at $37^{\circ} \mathrm{C}$ for 0,20 or 40 min. Immediately after the treatment, the cells were collected. Northern analysis was carried out using total RNA (10 $\mu \mathrm{g})$ prepared from the cells. Cloned cDNA of Japanese flounder HSC71 was digested by EcoRV (base pairs 1435 to 1440) and HindIII (2022-2027) (Fig. 1). The cDNA fragment (1438 to 2026 ) was used as the probe for hybridization. Hybridization was performed under the same conditions as described above.

\section{Staining RNA after Transfer to Filters}

As an internal control, RNA on the filter was stained after hybridization and exposure to X-ray film. The dried filter was soaked and stained in a solution of $0.2 \mathrm{M}$ sodium acetate $(\mathrm{pH} \mathrm{5.2)}$ and $0.2 \%$ methylene blue for $5 \mathrm{~min}$ at room temperature. The filter was rinsed in water for 10 $\min$.

\section{Results and Discussion}

\section{Cloning and Sequence of the Full-length cDNA for HSC7I}

An RT-PCR strategy for cloning a partial cDNA of Japanese flounder HSC71 successfully yielded a cDNA fragment of approximately $1090 \mathrm{bp}$. DNA sequencing analysis revealed that the fragment was homologous to rainbow trout HSC71 gene ${ }^{\text {() }}$ (data not shown). In order to obtain a full length cDNA of flounder HSC71, a flounder embryo cDNA library was screened with the PCR derived cDNA fragments as a probe. By screening of $10^{5}$ independent recombinant phages, seven positive clones were isolated. DNA sequencing analyses on both $5^{\prime}$ and $3^{\prime}$-end revealed that they encoded the same gene (data not shown). As a result, we determined the nucleotide sequences of the longest clone.

The cDNA of Japanese flounder HSC71 consists of 2242 nucleotides with poly (A) tail (Fig. 1). The in-frame stop codon in the 5' and 3' regions of the cDNA indicated that the clone contains the entire coding regions. The coding region includes 1950 nucleotides and corresponds to 650 amino acids with a predicted mass of $71.2 \mathrm{kDa}$.

Figure 2 compares the nucleotide sequence of HSC71 from Japanese flounder with those of other vertebrate. Japanese flounder HSC71 contains a EEVD (Glu-Glu-ValAsp) peptide motif at the C-terminal end which is the common feature of cytosolic HSC proteins in the HSP70 family. ${ }^{1)}$ Comparison with protein sequences in the SWISS-PROT database by FASTA indicated that Japanese flounder HSC71 is $96.0 \%, 93.9 \%, 93.5 \%$, and 93.4\% identical in primary structure to rainbow trout HSC71, ${ }^{6}$ bovine $\mathrm{HSC} 71,{ }^{8)}$ rat $\mathrm{HSC} 71,{ }^{9)}$ and human HSC $71,{ }^{7}$ respectively. Obviously the HSC71 amino acid sequence has been highly conserved in vertebrates. On the other hand, similarity to the heat inducible HSP70 is more divergent. For example, similarity to human $\mathrm{HSP}^{10}{ }^{10}$ amounts to $84.5 \%$ (Fig. 2).

\section{Constitutive Expression of Japanese Flounder HSC7I $m R N A$}

To investigate the expression pattern of Japanese flounder HSC71 mRNA, Northern blot analyses of total RNA from normal or heat-shocked cells were conducted (Fig. 3). The probe for hybridization was prepared from the cloned cDNA fragments (base pairs 1438 to 2026) that contained 3' untranslated regions (Fig. 1) to tighten the specific affinity on HSC71 mRNA rather than HSP70 mRNA. An approximate $2.2-\mathrm{kb}$ band was observed on RNA isolated from unheat shocked cells. No significant signal increase was observed after heat shock. Yamashita ${ }^{1)}$ reported the similar results on the platyfish HSC70, and also Zefarullah et al. on the rainbow trout HSC71. ${ }^{\text {() }}$ The structural similarities (Fig. 2) together with an identical mode of expression (Fig. 3) leave no doubts that the cloned cDNA described in this study represents a cDNA of heat shock cognate protein.

Analyses of the expression patterns of Japanese flounder HSC71 in embryos during development under normal and stress conditions are now in progress. cDNA cloning of Japanese flounder HSP70 and analyses of the expression patterns of HSP70 in embryos under normal and stress conditions are also now in progress.

Acknowledgments This study was supported in part by a Grant-in-Aid for Scientific Research from the Ministry of Education, Science, Sports and Culture of Japan and by a grant from the Research for the Future Program of the Japan Society for the Promotion of Science (97L00902).

\section{References}

1) M. Yamashita: Genes associated with stress response, in "Fish DNA" (ed. by T. Aoki, F. Takashima, and T. Hirano), Koseisya Koseikaku, Tokyo, 1997, pp. 219-243 (in Japanese).

2) K. B. Palter, M. Watanabe, L. Stinson, A. P. Mahowald, and E. A. Craig: Expression and localization of Drosophila melanogaster hsp70 cognate proteins. Mol. Cell. Biol., 6, 1187-1203 (1986).

3) E. A. Craig and K. Jacobsen: Mutations in cognate genes of Saccharomyces cerevisiae hsp70 result in reduced growth rates at low 
temperatures. Mol. Cell. Biol., 5, 3517-3524 (1985).

4) C. R. Brown, R. L. Martin, W. J. Hansen, R. P. Beckmann, and W. J. Welch: The constitutive and stress inducible forms of hsp70 exhibit functional similarities and interact with one another in an ATP-dependent fashion. J. Cell Biol., 120, 1101-1112 (1993).

5) T. Maniatis, E. F. Fritsch, and J. Sambrook: Molecular Cloning: A Laboratory Manual, 2nd edn. Cold Spring Harbor Laboratory Press, Cold Spring Harbor, NY, 1989.

6) M. Zafarullah, J. Wisniewski, N. W. Shworak, S. Schieman, S. Misra, and L. Gedamu: Molecular cloning and characterization of a constitutively expressed heat-shock-cognate hsc7l gene from rainbow trout. Eur. J. Biochem., 204, 893-900 (1992).
7) B. P. Dworniczak and M. E. Mirault: Structure and expression of a human gene coding a $71 \mathrm{kd}$ heat shock 'cognate' protein. Nuc. Acid Res., 15, 5181-5197 (1987).

8) C. D. Flaherty and D. Mckay: Nucleotide sequence of the CDNA of a bovine 70 kilodalton heat shock cognate protein. Nuc. Acid Res., 18, 5569 (1990).

9) K. O'Malley, A. Mauron, J. D. Barchas, and L. Kedes: Constitutively expressed rat mRNA encoding a 70-kilodalton heat-shocklike protein. Mol. Cell. Biol., 5, 3476-3483 (1985).

10) Hunt and R. I. Morimoto: Conserved features of eukaryotic hsp70 genes revealed by comparison with the nucleotide sequence of human hsp70. Proc. Natl. Acad. Sci. USA, 82, 6455-6459 (1985). 